\title{
Arterial Head Vascularization Cartographies of Normal Metencephalic Dogs Using Magnetic Resonance Angiography
}

\author{
OLIVIER D. JACQMOT, ${ }^{1 *}$ FREDERIC R. SNAPS, ${ }^{2}$ NATHALIE M. MAQUET, ${ }^{3}$ \\ MARIE PIERRE A. HEINEN, ${ }^{1}$ AND ANNICK E. GABRIEL ${ }^{1}$ \\ ${ }^{1}$ Department of Morphology and Pathology, Faculty of Veterinary Medicine, \\ University of Liege, Belgium \\ ${ }^{2}$ Department of Clinical Sciences, University of Liege, Belgium \\ ${ }^{3}$ Department of Clinical Sciences, Radiology and Medical Imaging, Faculty of Medicine, \\ University of Liege, Belgium
}

\begin{abstract}
The aim of our study was to establish head arterial cartographies-useful for the diagnosis of brain diseases leading to cerebral vascular modifications-by means of magnetic resonance angiography (MRA). Casts of the arterial vascular brain system were used to corroborate the MRA results as they can be easily rotated in nonvirtual three-dimensions and give an accurate view of the arteries calibre and origin. Two types of 3T MRA images were used: three-dimensional fast low-angle shot (3D-FLASH) acquisition sequenced every $20 \mathrm{~s}$, paired with injection of a paramagnetic contrast medium, and three-dimensional time-of-flight (3D-TOF) acquisition sequenced every $300 \mathrm{~s}$. 3D-FLASH acquisition gives very accurate images of the cerebral arteries and veins, but must be used with care in debilitated animals. $3 \mathrm{D}-\mathrm{TOF}$ acquisition is less accurate and gives only images of the main cerebral arteries without showing the venous system. It is, however, a viable diagnostic method for monitoring vascular lesions (e.g., cerebral hemorrhages). Anat Rec, 294:1834-1841, 2011. () 2011 Wiley-Liss, Inc.
\end{abstract}

\section{Key words: brain; vascularization; dog; magnetic resonance} angiography; anatomy

\begin{abstract}
Disturbances in the brain blood supply cause the brain abnormalities that underlie cerebrovascular diseases (Toole and Burrow, 1990). These diseases are a major cause of disability and the third leading cause of death in humans in developed countries. In comparison with humans, it is uncommon for animals to have cerebrovascular diseases, although the true incidence is unknown (Frankhauser et al., 1965). The most common types of cerebrovascular disease found in human patients, including infarctions, hemorrhages, and vascular anomalies, also occur in animals (Frankhauser et al., 1965; Wessmann et al., 2009). Interpreting the many physiological and pharmacological animal experiments requires accurate data on the vascular supply pattern of the brain, including information about the distribution, lengths, and internal calibres of the various major arteries. In our study, we focus only on distribution and localization of vessels.
\end{abstract}

The first techniques used for the study of brain vascularization were post mortem assessments like dissection and the "cast" technique. Preserved body material and previously fixed, isolated brains are inadequate for

Conflict of interest: None of the authors of this article has a financial or personal relationship with other people or organizations that could inappropriately influence or bias the content of the article.

*Correspondence to: Olivier D. Jacqmot, Faculté de Médecine Vétérinaire, Département de Morphologie et Pathologie, Service d'Anatomie, bât. B43, Boulevard de Colonster, 4000 Liege, Belgium. Fax: 32-4-3664076. E-mail: ojacqmot@ulg.ac.be

Received 30 January 2011; Accepted 29 July 2011

DOI 10.1002/ar.21479

Published online 1 October 2011 in Wiley Online Library (wileyonlinelibrary.com). 
modern research requirements, however, because they do not reflect physiological reality (Canossi et al., 1968). The injection of a synthetic resin into the vascular system of a recently living animal, followed by the dissolution of the organic material, is a more realistic and representative method (Tompsett and Wyke, 1961, 1969).

At present, the evolution of techniques allows us to visualize in three-dimensions the brain arterial vascularization in vivo by angiography, computed tomography scanning (CT-scan), and magnetic resonance imaging (MRI).

Arterial angiography, or arteriography, allows the radiological visualization of an arterial area by injection of a contrast agent via a catheter. Cerebral angiography is rarely performed in veterinary medicine because of the associated morbidity (Canossi et al., 1968; Dorn, 1972; Rising and Lewis, 1972) and difficulty in interpretation, even if it may be helpful when a vascular malformation is suspected (Thomas, 1996).

The MRI technique consists of measuring proton magnetization modification under the influence of two magnetic fields: a highly intense steady static magnetic field and a rotating electromagnetic field.

MRI and computed tomography can be used on dogs for the diagnosis of brain vascular pathologies such as hemorrhages (Jadhav et al., 2008, Wessmann et al., 2009), thromboses (Wessmann et al., 2009), cerebral vascular congenital malformations (Thomas et al., 1997), and intravascular lymphoma (Kent et al., 2001). Recently, they have also been used for brain vascularization study with a 1.5T MRI (Sager et al., 2009). In humans, studies are more complete (Jackowski et al., 2005), and diagnostic use is more frequent (Davis and Rumbaugh, 1967, Krainik et al., 2006).

We seek to establish a fuller arterial cartography of canine head vessels using 3T MRI. So far, previous studies (Sager et al., 2009) have used a 1.5T MRI, but because 3T MRI has an increased magnetic field strength, it should give better visualization of small intracranial vessels (Rodriguez et al., 2009). A cast technique and literature diagrams were used to compare and confirm the MRI pictures to establish head arterial cartography. We believe a vascular cartography could be useful in MRI for reference in accurately identifying the vessels of a given territory (e.g., caudal cerebral artery that vascularizes the brain caudal pole) and for making comparisons with the pictures obtained in neurological and/or vascular diseases. All information can be found in the literature, but much of it gives schematic information (Nickel et al., 1975; Anderson and Anderson, 1994; Barone, 1996) and an MRI picture is less readily interpreted.

Our study is limited to normal metencephalic dogs (i.e., neither brachycephalic nor dolichocephalic) to avoid differences arising from varying cranial morphologies; therefore, the head arterial cartography obtained will only be usable for head MRI of metencephalic dogs. We used the referential casts for adult dogs of different ages and weights to test whether the head vascular layout is influenced by such differences. According to prior descriptions (Nickel et al., 1975; Anderson and Anderson, 1994; Barone, 1996), all dogs are normal if the same structures are found at the same spots among all dogs.

\section{MATERIALS AND METHODS}

First, acrylic casts were made from the bodies of one group of healthy metencephalic dogs euthanized and given by the Belgian Animal Protection Society to compare and confirm further the MRI pictures. The protocol followed the code of ethics of University of Liege. The reference number from the Ethical Committee is 659 accepted on 5 July 2007.

The following materials for each canine body were used: $50 \mathrm{~g}$ of acryl powder (Flowing, Pouring Resin for Dentures, type II, class I, Biolux International) in a flask, $60 \mathrm{~mL}$ of methylmetacrylate (Flowing, Pouring Resin for Dentures, type II, class I, Biolux International) in another flask, two Pean clamps, two catheters, a 30 $\mathrm{mL}$ luer lock syringe, a manometer syringe (Sphere inflation device, Cook Medical) and a 36\% hydrochloric acid bath.

Five normal healthy dogs, ages 2-8 years old, were used: a female poodle, $10 \mathrm{~kg}$; a female Beagle, $15 \mathrm{~kg}$; a male Beagle, $16.5 \mathrm{~kg}$; a female Border Collie, $16 \mathrm{~kg}$; a female American Staff, $19.5 \mathrm{~kg}$. They were euthanized 2 $\mathrm{hr}$ before casting, and the two common carotids of each dog were isolated by an incision made at the neck right lateral medium third. Pean clamps were partially clamped on the isolated carotids as a catheter was inserted into each carotid.

The acryl and methylmetacrylate were homogenously mixed and used immediately because this mixture solidifies in 4 or $5 \mathrm{~min}$. The injection in one carotid by the 30 $\mathrm{mL}$ luer lock syringe was done under pressure so that the product could completely fill the vessels and flow out of the other carotid, which was then immediately clamped. The rest of the solution was manually injected with the manometer syringe at a 150 to $200 \mathrm{kPa}$ pressure. This pressure is much higher than normal physiological pressure $(17 \mathrm{kPa})$ to eject completely the blood from the major arteries as well as most of the small arteries since the dogs were not bled dry. An automatic injection pump and higher pressure were not used because they could have caused reflux into the veins, as well as bubbles, which would have weakened the "cast," or caused a leak by tearing a vessel. When the injection was complete, the entrance carotid was also clamped and the dog body was left for $1 \mathrm{hr}$ until the polymerization was complete.

The head was then removed and immersed in the $36 \%$ hydrochloric acid bath for at least 3 weeks so that the dissolution of the organic tissues was total. The cast obtained was rinsed out with clear water and dried.

Second, two MRI studies were performed using normal healthy Beagle dogs, six female and eight male, weighing between 10 and $16 \mathrm{~kg}$ and aged 2-4 years were fasted for $24 \mathrm{hr}$ before the experiment. The protocol followed the code of ethics of University of Liege. The reference number from the Ethical Committee is the same as mentioned previously (i.e., 659 accepted 5 July 2007). They were premedicated $20 \mathrm{~min}$ before the test with 200 $\mu \mathrm{g} / \mathrm{kg}$ body weight (BW) of butorphanol $10 \mathrm{mg} / \mathrm{mL}$ (Dolorex, Intervet) by intravenous (IV) injection. This was followed by an IV injection of $10 \mu \mathrm{g} / \mathrm{kg}$ BW of medetomidine $1 \mathrm{mg} / \mathrm{mL}$ (Domitor, Pfizer). The anesthesia time was equal to the duration of the experiment. The animals were awakened with an intramuscular injection of atipamezole $5 \mathrm{mg} / \mathrm{mL}$ (Antisedan, Pfizer) to antagonize the residual medetomidine.

This part of our study related to the visualization and determination of head vascularization by magnetic resonance angiography (MRA) using a 3T MRI unit (Siemens 
Magneto Trio). Two sequences were used: a three-dimensional (3D) T2-weighted fast low-angle shot (FLASH) gradient echo sequence requiring a contrast medium (or contrast-enhanced magnetic resonance angiography: CEMRA) and a time-of-flight (TOF) sequence.

A head-surface coil was used to improve the signal. The TOF acquisition needed long sequences (acquisition time [TA], $300 \mathrm{~s}$ ) and was based on enhanced inflowing blood without contrast agent. The thickness of the sections was from 0.5 to $0.7 \mathrm{~mm}$. The echo time (TE) was $3.69 \mathrm{~ms}$, the repetition time (TR) was 21 or $22 \mathrm{~s}$, the field of view (FOV) was 99 by $99 \mathrm{~mm}$ or 110 by $110 \mathrm{~mm}$ and the matrix was 240 by 500 .

For FLASH acquisition, a paramagnetic contrast medium was injected into the cephalic vein using a catheter. The contrast medium was a gadobutrol $(1 \mathrm{mmol} / \mathrm{mL}$; Gadovist, Schering Plough) bolus injected at a dose of $0.1 \mathrm{~mL} / \mathrm{kg}$ at $2 \mathrm{~mL} / \mathrm{s}$. The TA was $20 \mathrm{~s}$, and section thickness was $0.7 \mathrm{~mm}$. The acquisition was T2-weighted with a short flip angle of $20^{\circ}$. The TE was $1.33 \mathrm{~ms}$, the TR was $3.29 \mathrm{~ms}$, the FOV was 319 by $319 \mathrm{~mm}$ and the matrix was 230 by 320 . The post processing of the images was done using VB 15 software and a 3D maximum intensity projection (MIP) graphic card.

\section{RESULTS}

All anatomical terms comply with the WAVA Nomina Anatomica Veterinaria. We located and identified several main arteries on the casts using the literature: common carotid artery (arteria carotis communis), external carotid artery (a. carotis externa), internal carotid artery (a. carotis interna), basilar artery (a. basilaris), maxillary artery (a. maxillaris), and cerebral arterial circle (circulus arteriosus cerebri). Because casts could be handled as needed and examined in the lateral, medial and ventral planes, it was possible to have a good view of arterial courses. This was helpful in analyzing and corroborating the 3D Flash and 3D TOF MRA pictures. The casts showed no differences between individual animals. All the metencephalic dogs had the same cranial morphology (Figs. 1 and 2).

We measured the calibre of several arteries on one cast and in 3D Flash MRA pictures, and the results were equivalent: the calibre of the common carotid artery was $3.8 \mathrm{~mm}$ on the cast and $4 \mathrm{~mm}$ in the MRA picture; the calibre of the maxillary artery was $2.8 \mathrm{~mm}$ on the cast and $3 \mathrm{~mm}$ in the MRA picture; and the calibre of the basilar artery was $2.3 \mathrm{~mm}$ on the cast and $2.5 \mathrm{~mm}$ in the MRA picture. These results confirm that there were no major differences between metencephalic individuals despite the different weights and ages.

The 3D Flash acquisition MRA gave a very accurate image of the main arteries as well as intracranial and extracranial veins, venous sinuses and main brain plexus. The 3D TOF acquisition was less accurate and gave only the image of the main cerebral arteries, which were partly hidden by the venous sinuses and by the intracranial and extracranial venous plexus in the $3 \mathrm{D}$ Flash acquisition. Arteries and veins could be differentiated using the literature (e.g., the external jugular vein is more peripheral than the carotid artery, which is situated deep in the neck) and contrast differences (the contrast medium is at maximum concentration in the arteries so they appear whiter than veins in which a

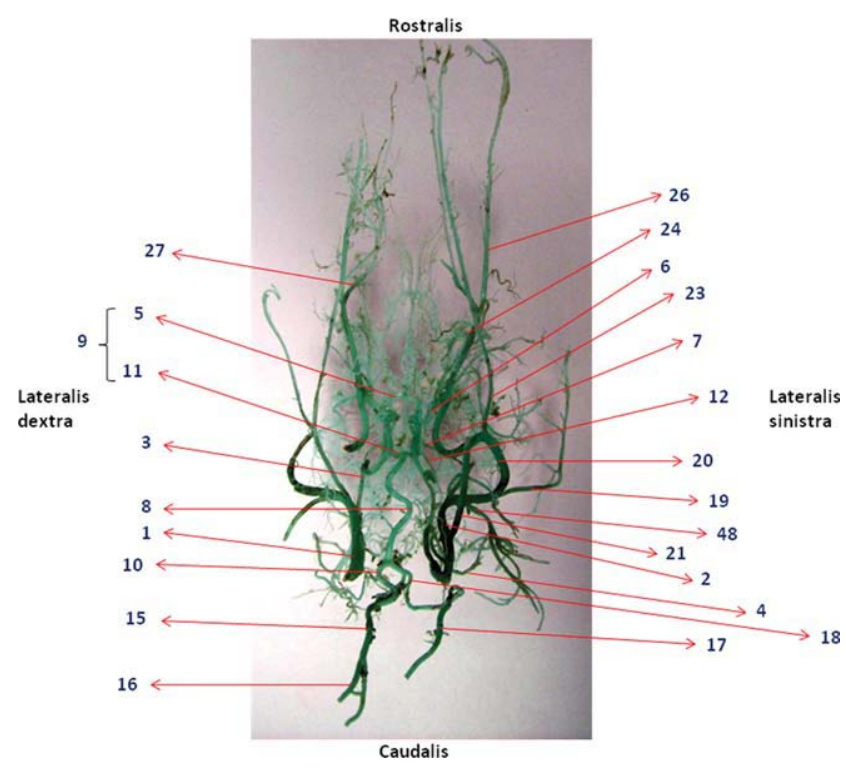

Fig. 1. Cast ventral view of dogs main head arteries. 1: Arteria (A.) carotis communis. 2: A. carotis externa. 3: A. carotis interna. 4: A. occipitalis. 5: A. cerebri rostralis. 6: Branch of A. cerebri media. 7: A. cerebri caudalis. 8: A. basilaris. 9: Circulus arteriosus cerebri. 10: Circulus arteriosus spinalis. 11: A. communicans caudalis. 12: A. cerebelli rostralis. 15: A. spinalis ventralis. 16: Ramus spinalis A. vertebralis. 17: Ramus anastomoticus $A$. vertebralis. 18: A. cerebrospinalis (A. vertebralis). 19: A. temporalis superficialis. 20: A. maxillaris. 21: A. lingualis. 23: A. alveolaris inferior. 24: A. ophtalmica externa. 26: A. infraorbitalis. 27: A. palatina descendens. 48: A. auricularis caudalis.

small quantity of contrast medium is present, giving the veins a grayish hue).

The TOF acquisition allowed us to visualize the following arteries (Figs. 3-5): common carotid artery (a. carotis communis), external carotid artery (a. carotis externa), internal carotid artery (a. carotis interna), occipital artery (a. occipitalis), rostral cerebral artery (a. cerebri rostralis), branch of middle cerebral artery (a. cerebri media), caudal cerebral artery (a. cerebri caudalis), basilar artery (a. basilaris), cerebral arterial circle (circulus arteriosus cerebri), spinal arterial circle (circulus arteriosus spinalis), caudal communicating artery (a. communicans caudalis), rostral cerebellar artery (a. cerebelli rostralis), ventral spinal artery (a. spinalis ventralis), spinal branch of vertebral artery (ramus spinalis a. vertebralis), anastomotic ramus of vertebral artery (ramus anastomoticus a. vertebralis), superficial temporal artery (a. temporalis superficialis), maxillary artery (a. maxillaris), lingual artery (a. lingualis), facial artery (a. facialis), inferior alveolar artery (a. alveolaris inferior), external ophthalmic artery (a. ophtalmica externa), infraorbital artery (a. infraorbitalis), descending palatine artery (a. palatina descendens), and dorsum sellae. The advantage of the Flash sequence lies in the fact that it gives a better spatial resolution and shows more arteries on top of the ones seen with the TOF sequence (Figs. 6 and 7), that is, cranial meningeal artery (a. meningea media, vertebral artery (a. vertebralis), cerebrospinal artery (a. cerebrospinalis), deep caudal temporal artery (a. temporalis profunda caudalis), minor palatine artery (a. palatina minor), and major palatine artery (a. 


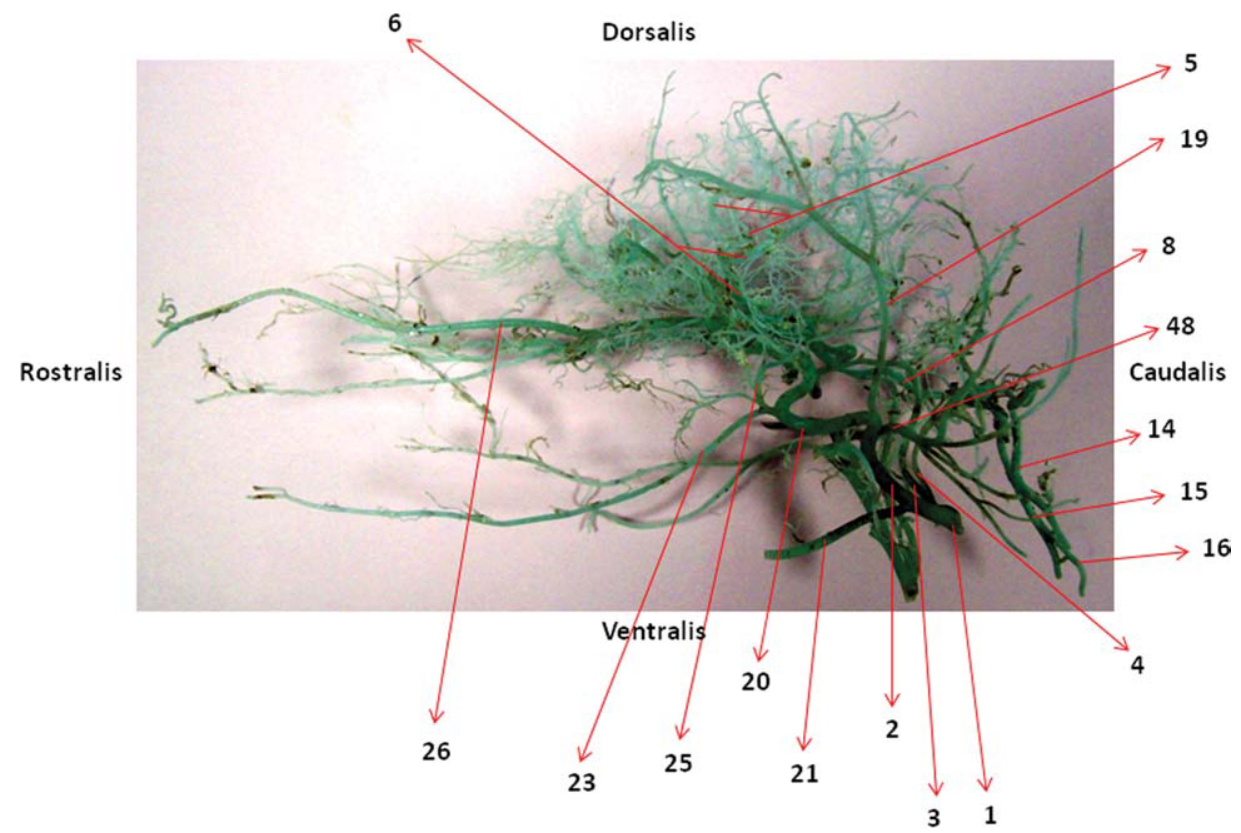

Fig. 2. Cast lateral view of dogs main head arteries. 1: A. carotis communis. 2: A. carotis externa. 3: A. carotis interna. 4: A. occipitalis. 5: A. cerebri rostralis. 6: Branch of A. cerebri media. 8: A. basilaris. 14: A. vertebralis. 15: A. spinalis ventralis. 16: Ramus spinalis. 19: A. temporalis superficialis. 20: A. maxillaris. 21: A. lingualis. 23: A. alveolaris inferior. 25: A. temporalis profunda caudalis. 26: A. infraorbitalis. 48: A. auricularis caudalis.

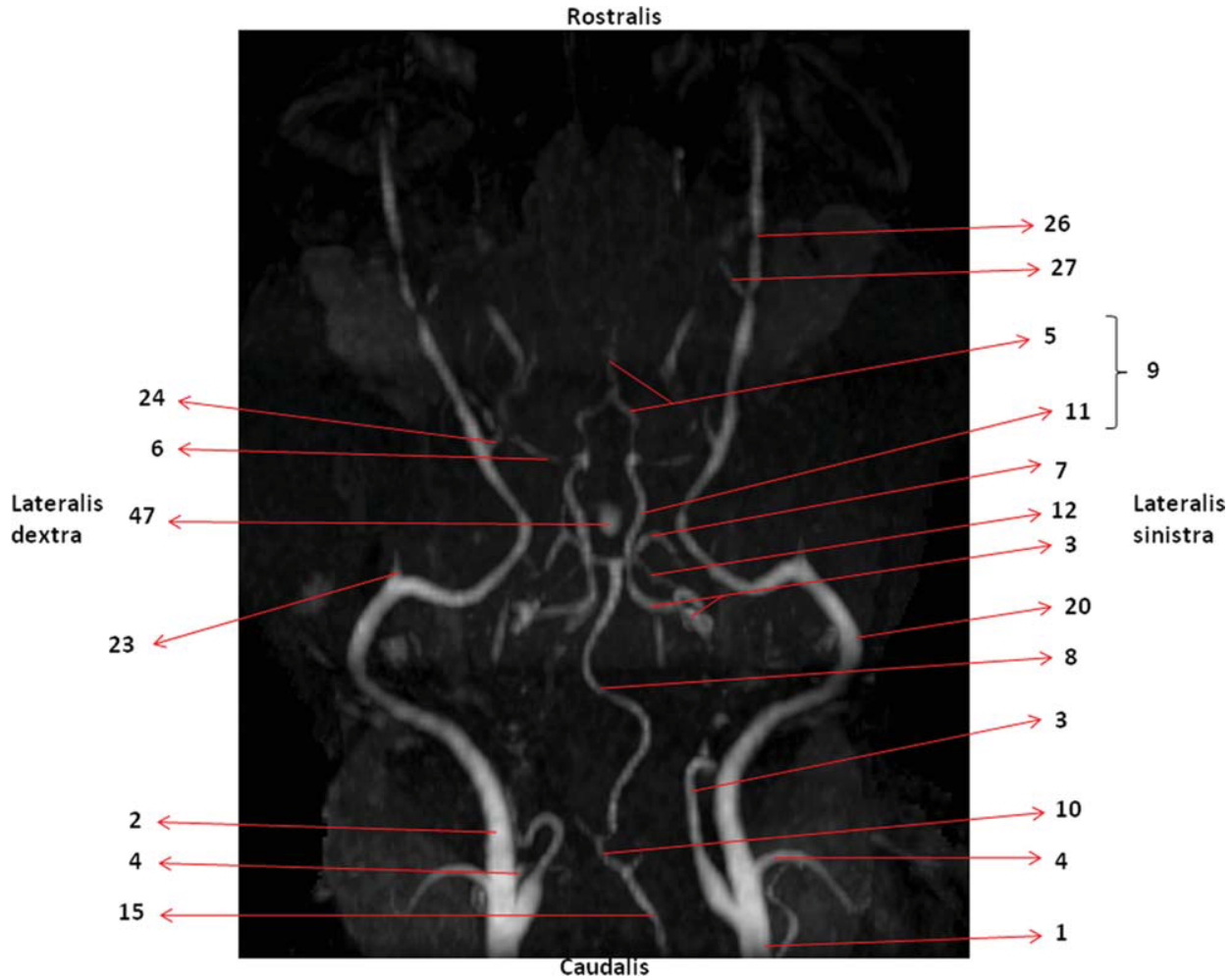

Fig. 3. TOF MRA acquisition ventral view of dogs head. Time of echo (TE): $3.69 \mathrm{~ms}$; Time of repetition (TR): $21 \mathrm{~s}$; Field of view (FOV): 99 by $99 \mathrm{~mm}$. 1: A. carotis communis. 2: A. carotis externa. 3: A. carotis interna. 4: A. occipitalis. 5: A. cerebri rostralis. 6: Branch of A. cerebri media. 7: A. cerebri caudalis. 8: A. basilaris. 9: Circulus arteriosus cerebri. 10: Circulus arteriosus spinalis. 11: A. communicans caudalis. 12: A. cerebelli rostralis. 15: A. spinalis ventralis. 20: A. maxillaris. 23: A. alveolaris inferior. 24: A. ophtalmica externa. 26: A. infraorbitalis 27: A. palatina descendens. 47: Dorsum sellae. 


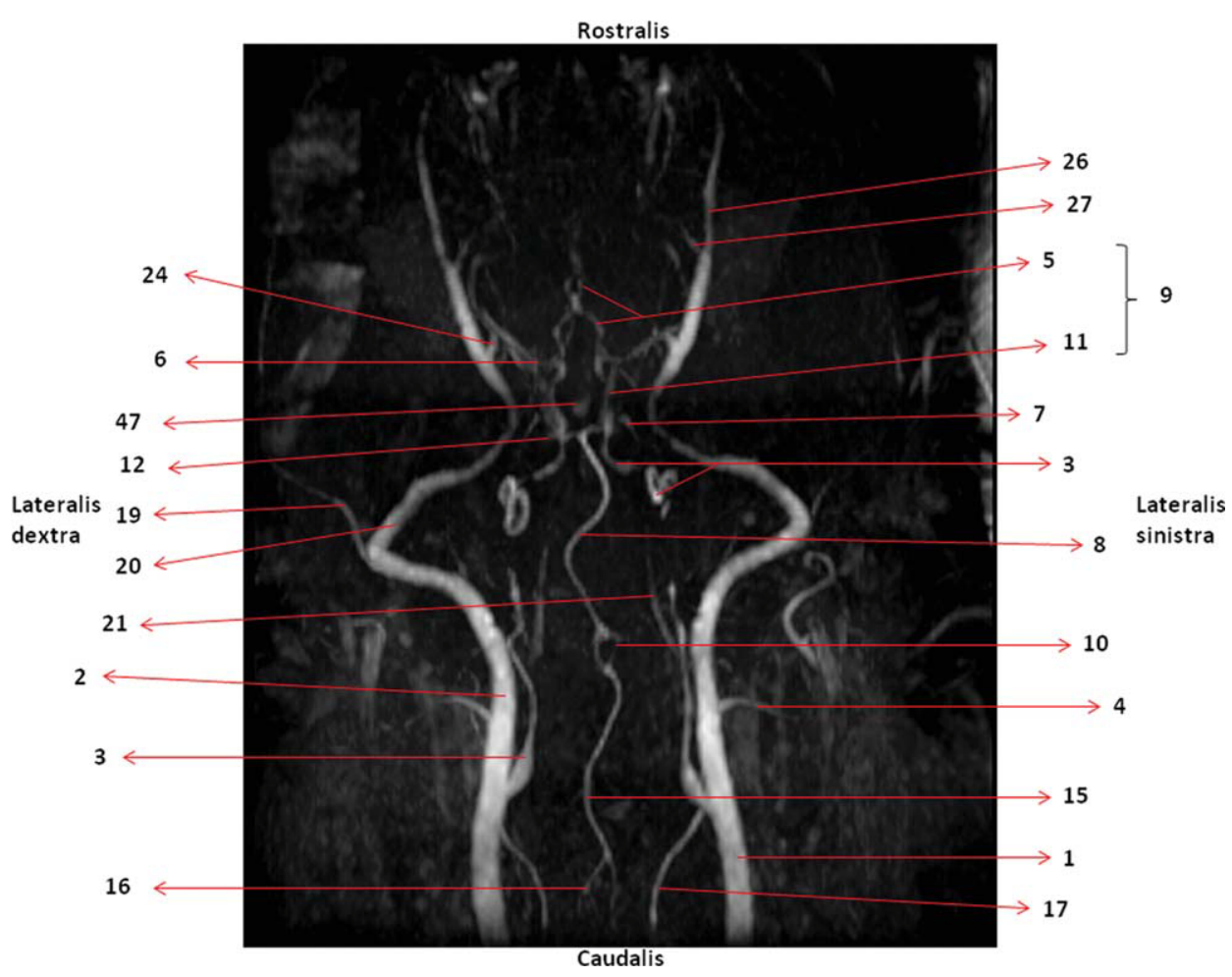

Fig. 4. TOF MRA acquisition ventral view of dogs head. Time of echo (TE): $3.69 \mathrm{~ms}$; Time of repetition (TR): $22 \mathrm{~s}$; Field of view (FOV): 110 by $110 \mathrm{~mm}$. 1: A. Carotis communis. 2: A. carotis externa. 3: A. carotis interna. 4: A. occipitalis. 5: A. cerebri rostralis. 6: Branch of A. cerebri media. 7: A. cerebri caudalis. 8: A. basilaris. 9: Circulus arteriosus cerebri. 10: Circulus arteriosus spinalis. 11: A. communicans cau-

palatina major). It also showed the following venous plexus and sinuses and veins: external jugular vein (v. jugularis externa), lingo facial vein (v. lingofacialis), facial vein (v. facialis), angularis oculi vein (v. angularis oculi), dorsal external ophthalmic vein (v. ophtalmica externa dorsalis), ventral external ophthalmic vein (v. ophtalmica externa ventralis), maxillary vein (v. retromandibularis [maxillaris]), caudal auricular vein (v. auricularis caudalis), ophthalmic venous plexus (plexus ophtalmicus), interarcuate branch (v. basivertebralis), ventral internal vertebral venous plexus (plexus vertebralis internus ventralis), cavernous sinus (sinus cavernosus), rostral intercavernous sinus (sinus intercavernosus rostralis), caudal intercavernous sinus (sinus intercavernosus caudalis), ventral petrosal sinus (sinus petrosus ventralis), and basilar sinus (sinus basilaris).

\section{DISCUSSION}

The aim of our study was to establish two-dimensional cartography of normal metencephalic head arteries in dogs using 3T MRI rather than $1.5 \mathrm{~T}$ MRI as in previous studies (Sager et al., 2009). Three-tesla pictures have better resolution (Rodriguez et al., 2009) than 1.5T, and we were able to identify many more arteries ( 29 vs. 9 in the Rodriguez et al. study) and 47 vessels in total. In fact, we created two cartographies-one with the TOF dalis. 12: A. cerebelli rostralis. 15: A. spinalis ventralis. 16: Ramus spinalis A. vertebralis. 17: Ramus anastomoticus A. vertebralis. 19: A. temporalis superficialis. 20: A. maxillaris. 21: A. lingualis. 24: A. ophtalmica externa. 26: A. infraorbitalis. 27: A. palatina descendens. 47: Dorsum sellae.

MRA and the other with the Flash MRA-for metencephalic dogs.

3D Flash acquisition MRA or CE-MRA, in which a contrast medium is injected into the cephalic vein, allows visualization of tortuous vessels. It gives excellent spatial resolution (i.e., ability to distinguish small vessels in close proximity) thanks to the MIP process and the contrast medium, but its major drawback is that some intracranial arteries are hidden by the cranial venous sinuses and venous plexus. Even by rotation of the 3D Flash pictures after the MIP process, some arteries are not displayed because of the superimposition of venous structures (e.g., cavernous sinus, rostral intercavernous sinus and caudal intercavernous sinus hide parts of the internal carotid artery, cerebral arterial circle and the anastomosis of basilar artery with the cerebral arterial circle). Therefore, the $3 \mathrm{D}$ TOF acquisition is essential for visualizing the intracranialand extracranial arteries without the venous system, but it has the disadvantage of less accurate pictures than the Flash acquisition.

The Flash acquisition MRA, which requires the use of a contrast medium, must be used with care in debilitated animals. For instance, life-threatening contrastinduced nephropathy may occur in animals with moderate to severe chronic kidney disease (Hasebroock and Serkova, 2009). In cases of debilitated animals for which Flash acquisition MRA would pose too much risk, TOF 


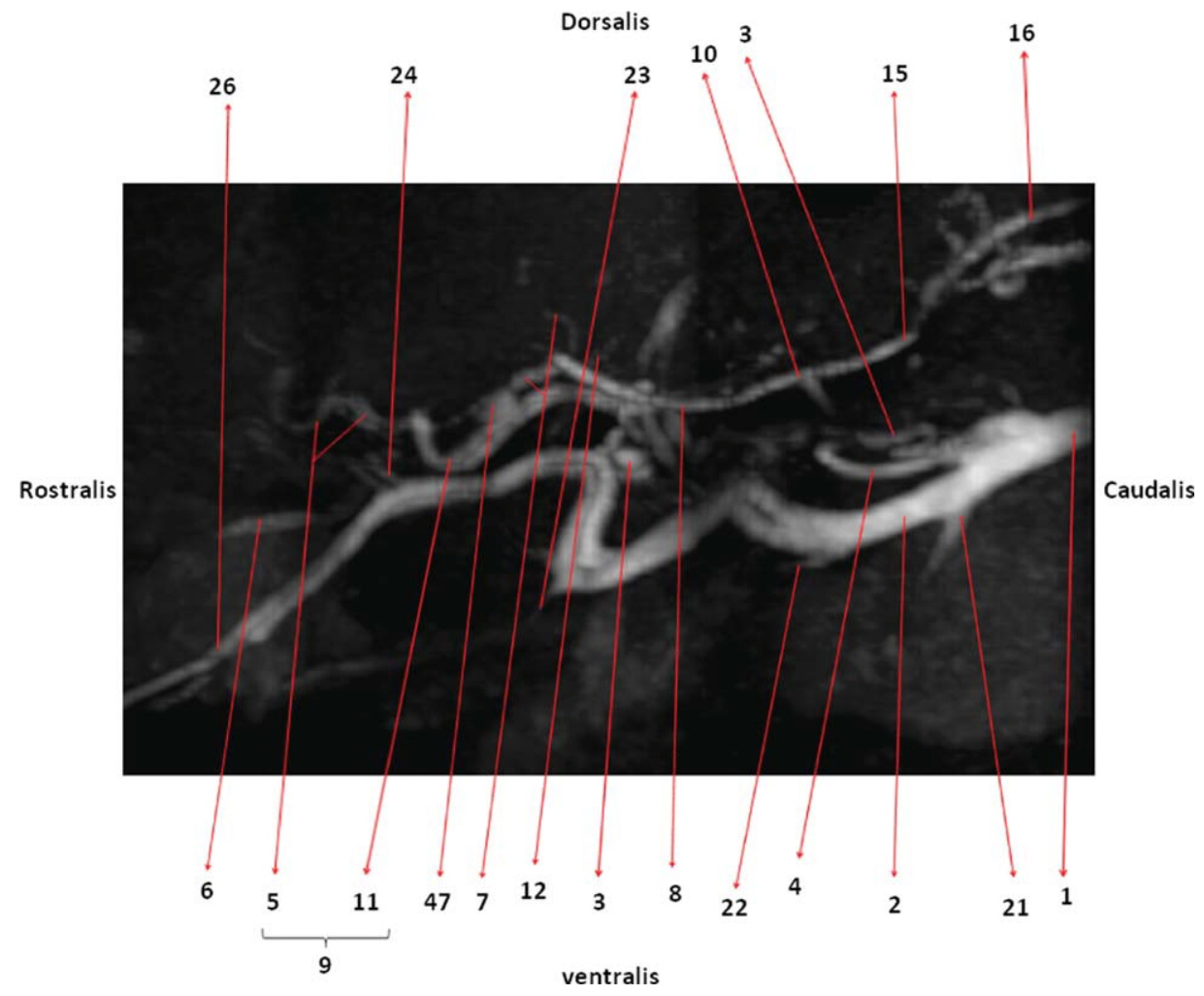

Fig. 5. TOF MRA acquisition lateral view of dogs head. Time of echo (TE): $3.69 \mathrm{~ms}$; Time of repetition (TR): $21 \mathrm{~s}$; Field of view (FOV): 99 by $99 \mathrm{~mm}$. 1: Carotis communis. 2: Carotis externa. 3: Carotis interna. 4: A. occipitalis. 5: A. cerebri rostralis. 6: Branch of A. cerebri media. 7: A. cerebri caudalis. 8: A. basilaris. 9: Circulus arteriosus cer- ebri. 10: Circulus arteriosus spinalis. 11: A. communicans caudalis. 12: A. cerebelli rostralis. 15: A. spinalis ventralis. 16: Ramus spinalis $A$. vertebralis. 21: A. lingualis. 22: A. facialis. 23: A. alveolaris inferior. 24: A. ophtalmica externa. 26: A. infraorbitalis. 47: Dorsum sellae. acquisition is the better choice even if the pictures are less accurate.

The 3T-MRI images and thus the two-dimensional cartographies of normal metencephalic head arteries in dogs obtained in our study are more appropriate for research purposes than for diagnosis as actually the trend is to buy low-field MRI machines for diagnostic purposes in veterinary medicine because of their high purchase and maintenance costs. However, these diagnostic methods-Flash and TOF MRA - are led to be used more frequently in clinical cases in the future. In our opinion, vascular MRI two-dimensional cartography could be helpful in MRI for accurately identifying the vessels of a given territory because difficulties may arise in differentiating between minor arterial and venous vessels. This information can be found in the literature, but the schematic information may not be very helpful in interpreting an MRI picture.

To create the TOF MRA and the Flash MRA cartographies we referred to previous references (Nickel et al., 1975; Anderson and Anderson, 1994; Barone, 1996) and used a cast technique. The literature provided a schematic interpretation, and the casts furnished a 3D interpretation of the arterial courses. A cast is a useful tool because it can be easily rotated in three dimensions, and it gives an accurate view of the arteries' calibre and origin. All the metencephalic dogs in our study had the same cranial morphology, and we noticed no differences between individuals of different ages and weights.

Until a few years ago, the diagnosis of vascular brain diseases of dogs were made post mortem, so no treatments were proposed. With the use of techniques such as X-ray angiography, digital subtraction angiography, computed tomography angiography (CTA) and MRA, clinicians have the opportunity to study the arterial vasculature of the normal canine brain and are able to diagnose brain diseases accompanied by modified brain vascularization, such as intravascular cerebral lymphomas (Kent et al., 2001), thromboses (Wessmann et al., 2009), brain hemorrhages (Jadhav et al., 2008; Wessmann et al., 2009), and cerebral vascular malformations and their consequences (Thomas et al., 1997). In punctiform lesions, an arterial cartography could be useful to determine which artery is involved and thus which territory is injured. This allows the clinicians to select the most appropriate treatment option and to know if it will lead to a fatal issue.

According to some authors (Davis and Rumbaugh, 1967; Dorn, 1972; Perry and Lowrie, 1993) the use of conventional x-ray angiography in veterinary medicine is associated with technical difficulties and is impaired by the layout of the intracranial vessels. When contrast medium is injected into the common carotid or the external carotid artery, pictures of the intracranialand 


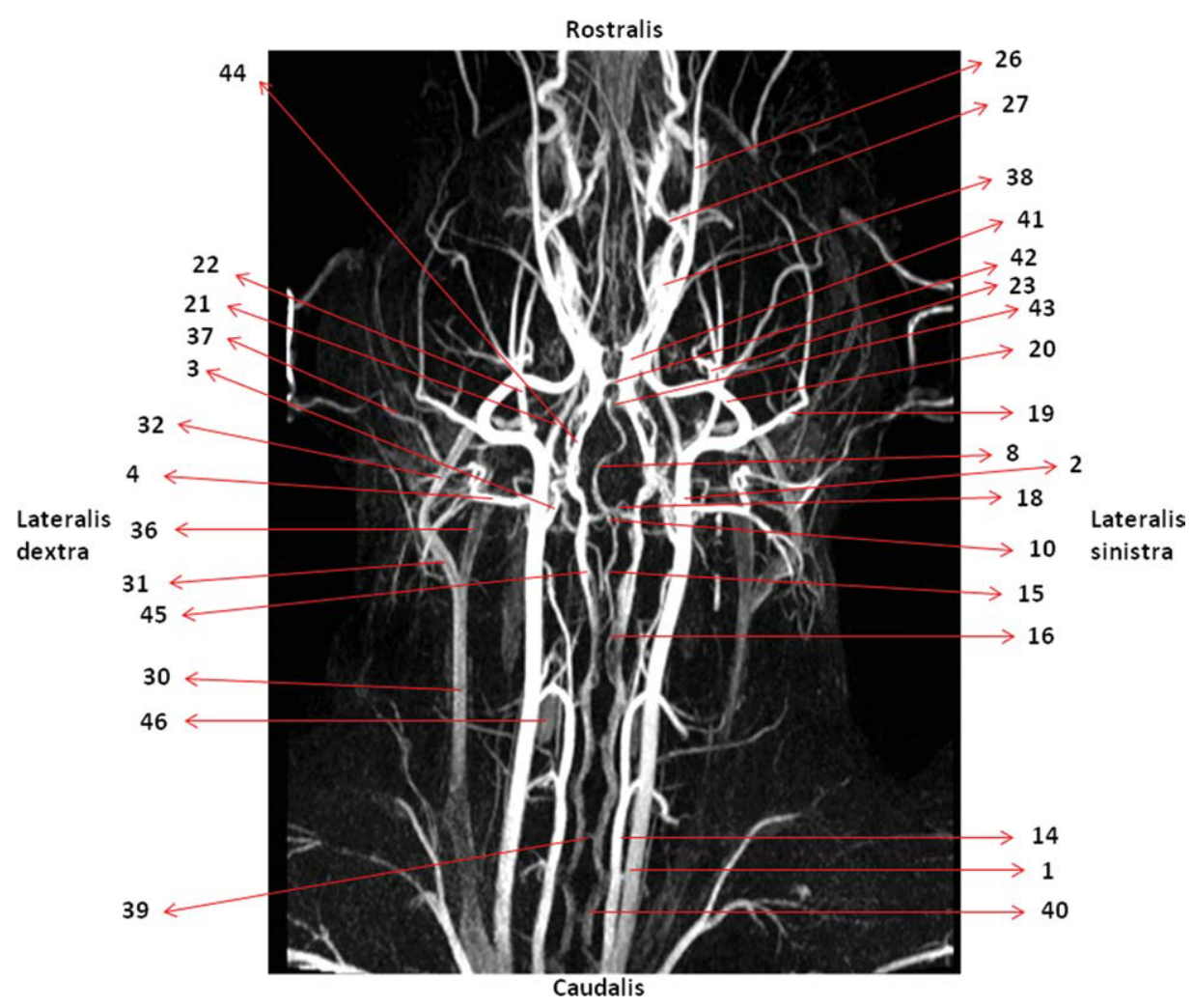

Fig. 6. FLASH MRA acquisition ventral view of dog head. Time of echo (TE): $1.33 \mathrm{~ms}$; Time of repetition (TR): $3.29 \mathrm{~ms}$; Field of view (FOV): 319 by $319 \mathrm{~mm}$. 1: A. carotis communis. 2: A. carotis externa. 3: A. carotis interna. 4: A. occipitalis. 8: A. basilaris. 10: Circulus arteriosus spinalis. 14: A. vertebralis. 15: A. spinalis ventralis. 16: Ramus spinalis A. vertebralis. 18: A. cerebrospinalis (A vertebralis). 19: A. temporalis superficialis. 20: A. maxillaris. 21: A. lingualis. 22: A. facia-

lis. 23: A. alveolaris inferior. 26: A. infraorbitalis. 27: A. palatina descendens. 30: Vena (V.) jugularis externa. 31: V. lingofacialis. 32: V. facialis. 36: V. maxillaris (retromandibularis). 37: V. auricularis caudalis. 38: Plexus ophtalmicus. 39: V. basivertebralis. 40: Plexus vertebralis internus ventralis. 41: Sinus cavernosus. 42: Sinus intercavernosus rostralis. 43: Sinus intercavernosus caudalis. 44: Sinus petrosus ventralis. 45: Sinus basilaris. 46: Glandula thyroidea.

Dorsalis

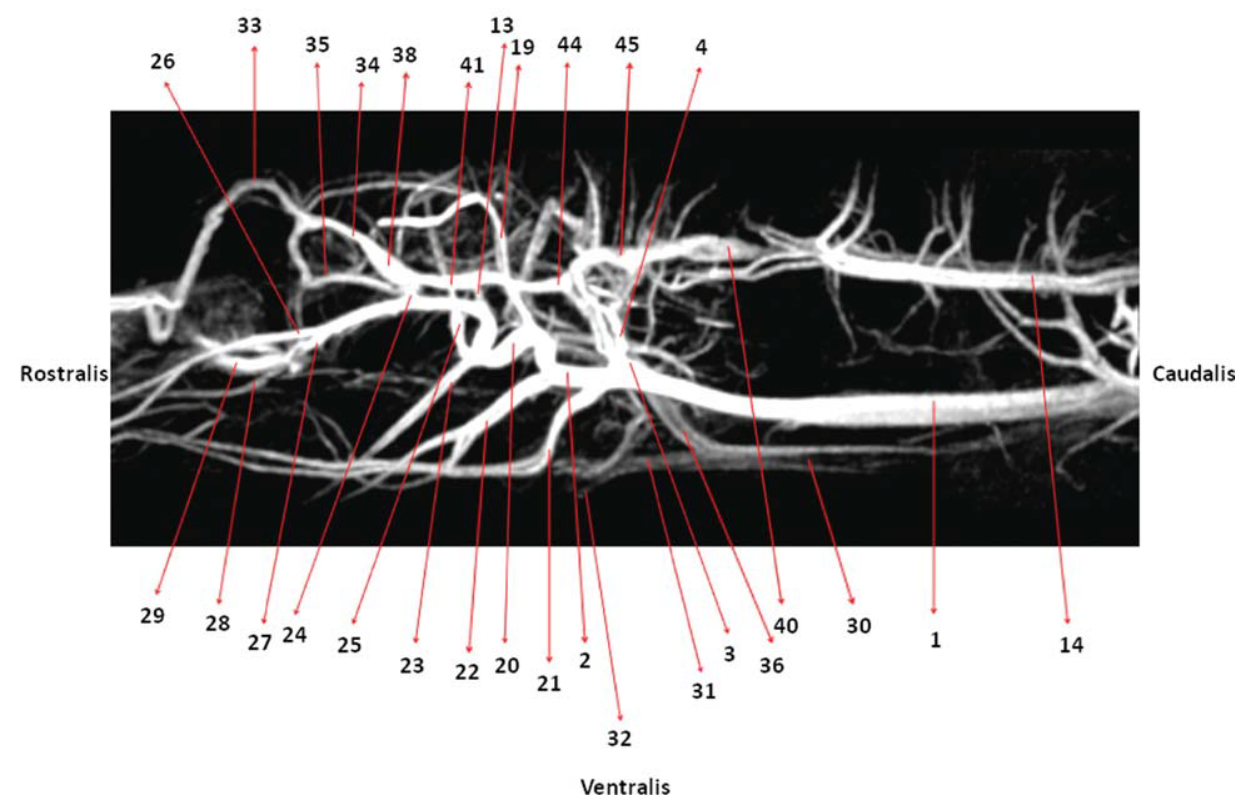

Fig. 7. FLASH MRA acquisition lateral view of dogs head. Time of echo (TE): $1.33 \mathrm{~ms}$; Time of repetition (TR): $3.29 \mathrm{~ms}$; Field of view (FOV): 319 by $319 \mathrm{~mm}$. 1: A. carotis communis. 2: A. carotis externa. 3: A. carotis interna. 4: A. occipitalis. 13: A. meningea media. 14: A. vertebralis. 19: A. temporalis superficialis. 20: A. maxillaris. 21: A. lingualis. 22: A. facialis. 23: A. alveolaris inferior. 24: A. ophtalmica externa. 25: A. temporalis profunda caudalis. 26: A. infraorbitalis. 27:

A. palatina descendens. 28: A. palatina minor. 29: A. palatina major 30: V. jugularis externa. 31: V. lingofacialis. 32: V. facialis. 33: V. angularis oculi. 34: V. ophtalmica externa dorsalis. 35: V. ophtalmica externa ventralis. 36: V. maxillaris (retromandibularis). 38: Plexus ophtalmicus. 40: Plexus vertebralis internus ventralis. 41: Sinus cavernosus. 44: Sinus petrosus ventralis. 45 : Sinus basilaris. 
extracranial vessels are superimposed. The resultant image quality is rather poor with this technique, and the neurological risks are significant (Davis and Rumbaugh, 1967; Dorn, 1972; Rising and Lewis, 1972; Sager et al., 2009).

The CTA technique, using both X-rays and a computer to obtain pictures in sections, and 3D Flash acquisition MRA have the same drawbacks as the x-ray technique in debilitated animals due to the iodated contrast medium (Hasebroock and Serkova, 2009). However, they have the advantage of providing $3 \mathrm{D}$ pictures and therefore represent how the vessels are positioned in relation to one another.

In summary, 3D TOF acquisition MRA technique is a rapid noninvasive technique, which can be used as a diagnostic method to evaluate intracranial arteries as small as $0.4 \mathrm{~mm}$ (Rodriguez et al., 2009). It is a good diagnostic tool for monitoring lesions such as cerebral hemorrhage in debilitated animals for which other methods must be used with care.

\section{CONCLUSIONS}

In human medicine, MRA has long been used for the diagnosis of vascular pathologies, allowing clinicians and researchers to find the best treatments. The MRA is a rapid and noninvasive technique, which can be used in dogs as a diagnostic method to evaluate intracranial arteries as small as $0.4 \mathrm{~mm}$ in diseases that lead to cerebral vascular modifications. TOF MRA cartography and Flash MRA cartography for metencephalic dogs should be helpful to clinicians for recognizing the vessels of a given territory.

\section{ACKNOWLEDGEMENTS}

Bruno Weckman and Olivier Lemaître (technicians in anatomy) helped prepare dogs and Dimitri Hayes (technician for the scan machine at the CHU of Liege) helped us in using the scan machine.

\section{LITERATURE CITED}

Anderson WD, Anderson BG. 1994. Head. In: Anderson WD, Anderson BG, editors. Atlas of canine anatomy. Philadelphia: Lea and Febiger. p 91-132, 188-207.

Barone R. 1996. Artères de la tête et du cou. In: Barone R, editor Anatomie comparée des mammifères domestiques, Tome 5: Angiologie. Paris: Vigot Frères. p 199-207, 223, 469-471.

Canossi GC, Dardari M, Cortesi N, Brunelli B, Pasquinelli C. 1968. Etude particulière de la tête. In: Canossi GC, editor. Anatomie angiographique du chien. Paris: Vigot Frères. p 21-25.
Davis DO, Rumbaugh CL. 1967. Cerebral angiography in the dog. Method for consistent results. Invest Radiol 2:323-325.

Dorn A. 1972. A standard technique for canine cerebral angiography. J Am Vet Med Assoc 161:1669-1675.

Frankhauser R, Luginbuhl H, McGrath JT. 1965. Cerebrovascular disease in various animal species. Ann N Y Acad Sci 127:817-859.

Hasebroock KM, Serkova NJ. 2009. Toxicity of MRI and CT contrast agents. Expert Opinion Drug Metab Toxicol 5:403-416.

Jackowski C, Sonnenschein M, Thali MJ, Aghayev E, von Allmen G, Yen K, Dirnhofer R, Vock P. 2005. Virtopsy: Postmortem minimally invasive angiography using cross section techniquesimplementation and preliminary results. J Forensic Sci 50:11751186.

Jadhav V, Sugawara T, Zhang J, Jacobson P, Obenaus A. 2008. Magnetic resonance imaging detects and predicts early brain injury after subarachnoid hemorrhage in a canine experimental model. J Neurotrauma 25:1099-1106.

Kent M, DeLahunta A, Tidwell AS. 2001. MR imaging findings in a dog with intravascular lymphoma in the brain. Vet Radiol Ultrasound 42:504-510.

Krainik A, Rubin C, Grand S, David O, Baciu M, Jaillard A, Troprès I, Lamalle L, Duffau H, Le Bas JF, Segebarth C, Lehéricy S. 2006. L’imagerie par résonnance magnétique cérébrale fonctionnelle en pratique clinique. J Radiol 87:607-617.

Nickel R, Schummer A, Seiferle E. 1975. Zentralnervensystem: Gefäßversorgung von Rückenmark und Gerhirn. In: Nickel R, Schummer A, Seiferle E, editors. Lehrbuch der Anatomie der Haustiere, Band IV: Nervensystem, Sinnesorgane, Endokrine Drüsen. Berlin, Hamburg: Verlag Paul Parey. p 174-188.

Perry RL, Lowrie CT. 1993. Selected contrast studies, portal venography and cerebral angiography. Vet Clin North Am Small Anim Pract 23:331-343.

Rising JL, Lewis RE. 1972. Femorovertebral cerebral angiography in the dog. Am J Vet Res 33:665-676.

Rodriguez D, Rylander H, Vigen KK, Adams WM. 2009. Influence of field strength on intracranial vessel conspicuity in canine magnetic resonance angiography. Vet Radiol Ultrasound 50:477-482.

Sager M, Assheuer J, Trümmler H, Moormann K. 2009. Contrastenhanced magnetic resonance angiography (CE-MRA) of intraand extra-cranial vessels in dogs. Vet J 179:92-100.

Thomas WB. 1996. Cerebrovascular disease. Vet Clin North Am Small Anim Pract 26:925-943.

Thomas WB, Adams WH, McGavin MD, Gompf RE. 1997. Magnetic resonance imaging appearance of intracranial hemorrhage secondary to cerebral vascular malformation in a dog. Vet Radiol Ultrasound 38:371-375.

Tompsett DH, Wyke BD. 1961. Comparative analysis of the cerebral arterial system in laboratory animals and man. J Physiol 156:23.

Tompsett DH, Wyke BD. 1969. A method for preparing corrosion casts of the cerebral arterial system of laboratory animals in the comparative study of the cerebral vascular system. Acta Anat 72:460-475.

Toole JF, Burrow DD. 1990. Pathophysiology and clinical evaluation of ischemic vascular disease. In: Youmans JR, editor. Neurological Surgery, 3rd ed. Philadelphia: WB. Saunders. p 1463-1515.

Wessmann A, Chandler K, Garosi L. 2009. Ischaemic and haemorrhagic stroke in the dog. Vet J 180:290-303. 